\title{
Examples of bosonic de Finetti states over finite dimensional Hilbert spaces
}

\author{
Alex D. Gottlieb
}

\begin{abstract}
According to the Quantum de Finetti Theorem, locally normal infinite particle states with Bose-Einstein symmetry can be represented as mixtures of infinite tensor powers of vector states. This note presents examples of infinite-particle states with Bose-Einstein symmetry that arise as limits of Gibbs ensembles on finite dimensional spaces, and displays their de Finetti representations. We consider Gibbs ensembles for systems of bosons in a finite dimensional setting and discover limits as the number of particles tends to infinity, provided the temperature is scaled in proportion to particle number.
\end{abstract}

\section{Introduction}

According to the Quantum de Finetti Theorem [2], locally normal infiniteparticle states with Bose-Einstein symmetry can be represented as mixtures of infinite tensor powers of vector states. This note presents examples of infinite-particle states with Bose-Einstein symmetry that arise as limits of Gibbs ensembles on finite dimensional spaces, and displays their de Finetti representations.

The central example is as follows. If the single-particle Hilbert space $\mathcal{H}$ is finite dimensional, the projector onto the symmetric subspace of the $n$ particle space can be normalized, and this defines the infinite-temperature ensemble for $n$ bosons with single-particle space $\mathcal{H}$. For each fixed $m \in \mathbb{N}$, the $m$-particle reduced density operators under the $n$-boson infinite-temperature ensembles converge, as $n$ tends to infinity, to the density operator describing the $m$-particle statistics under a certain bosonic infinite-particle state $\omega_{0}$. The infinite-particle state $\omega_{0}$ has a de Finetti representation as a mixture 
of infinite tensor powers of vector states $P_{\mathrm{v}}$, where $\mathrm{v}$ is a unit vector and $P_{\mathrm{v}}=|\mathrm{v}\rangle\langle\mathrm{v}|$ denotes the projector onto the span of $\mathrm{v}$. In the de Finetti mixture for $\omega_{0}$, the weight of the tensor power state $P_{\mathrm{v}}{ }^{\otimes \infty}$ is the probability density for $\mathrm{v} \in \mathcal{H} \cong \mathbb{C}^{d+1}$ to equal

$$
\mathrm{v}(p, \theta)=\left(e^{i \theta_{0}} \sqrt{p_{0}}, e^{i \theta_{1}} \sqrt{p_{1}}, \ldots, e^{i \theta_{d}} \sqrt{p_{d}}\right)
$$

when $p=\left(p_{0}, p_{1}, \ldots, p_{d}\right)$ is sampled uniformly from the $d$-dimensional simplex $\Delta_{d}$ and the phase angles $\theta_{i}$ in $\theta=\left(\theta_{0}, \theta_{1}, \ldots, \theta_{d}\right)$ are each sampled uniformly from $[0,2 \pi)$, independently of one another and of $p$. Thus the infinite-particle state $\omega_{0}$ corresponds to the uniform probability measure on $\Delta_{d} \times[0,2 \pi)^{d+1}$.

Similar limits are obtained for finite temperature Gibbs ensembles, provided the temperature is scaled properly. Suppose $H$ is a Hermitian operator on the single-particle space $\mathcal{H} \cong \mathbb{C}^{d+1}$ and $\Gamma_{n}(\beta)$ denotes the Gibbs canonical ensemble for $n$ noninteracting bosons with single-particle Hamiltonian $H$ at inverse temperature $\beta$. Then, as $n$ tends to infinity, the $m$-particle reduced density operators under $\Gamma_{n}(\beta / n)$ converge to the $m$-particle density of a certain bosonic infinite-particle state $\omega_{\beta}$. The infinite-particle state $\omega_{\beta}$ is an average of states $P_{\mathrm{v}}^{\otimes \infty}$ with respect to the probability density on $\Delta_{d} \times[0,2 \pi)^{d+1}$ that minimizes the "free energy"

$$
\int_{[0,2 \pi)^{d+1}} \int_{\Delta_{d}}\langle\mathrm{v}, H \mathrm{v}\rangle f(p, \theta) d p d \theta+\frac{1}{\beta} \int_{[0,2 \pi)^{d+1}} \int_{\Delta_{d}} f(p, \theta) \ln f(p, \theta) d p d \theta,
$$

where $\mathrm{v}=\mathrm{v}(p, \theta)$ is as in (11). We obtain similar results for bosons with "mean field" interactions, but again we must scale temperature in proportion to the number of particles. This stands in contrast to the analogous mean field limits for distinguishable particles, which are obtained without any peculiar scaling of temperature [1].

The physical relevance of these facts is limited. On the one hand, they concern limits of canonical ensembles, which are appropriate when the number of bosons is fixed, and therefore not appropriate for massless bosons (e.g., photons). On the other hand, massive bosons inhabit infinite dimensional Hilbert spaces, so to speak, whereas our results concern finite dimensional Hilbert spaces. However, the sort of ensemble we study is appropriate for (noninteracting) systems of $n$ material bosons in thermal equilibrium, in case it is known that every one of these bosons is trapped in a potential well of 
depth E. The statistical state of that system would be a conditional Gibbs ensemble, supported on the finite dimensional Hilbert space spanned by the symmetrized products of trapped (bound) states. Only noninteracting systems of trapped bosons are considered, because the conditional Gibbs ensemble only makes sense if the Hamiltonian of the system commutes with the observable that every particle is trapped.

Our results are presented in Section [3, after a quick review of the Quantum de Finetti Theorem in the next section.

\section{The Quantum de Finetti Theorem}

Let $\mathcal{H}$ be Hilbert space (which we will call the single-particle Hilbert space) and let $\mathcal{H}^{\otimes n}$ denote the $n$-fold tensor power of $\mathcal{H}$ (the $n$-particle Hilbert space). When $\pi$ denotes a permutation of $\{1,2, \ldots, n\}$, let $U_{\pi}$ denote the unitary "permutation" operator on $\mathcal{H}^{\otimes n}$ defined by

$$
U_{\pi}\left(x_{1} \otimes x_{2} \otimes \cdots \otimes x_{n}\right)=x_{\pi(1)} \otimes x_{\pi(2)} \otimes \cdots \otimes x_{\pi(n)} .
$$

For each $n \in \mathbb{N}$ let $D_{n}$ be a density operator on the $n$-particle Hilbert space $\mathcal{H}^{\otimes n}$, the $n$-fold tensor power of $\mathcal{H}$. We want the density operators $D_{n}$ to be symmetric, and we assume

(A) for all $n$, the density operator $D_{n}$ commutes with any permutation operator $U_{\pi}$ on $\mathcal{H}^{\otimes n}$.

We are especially interested here in systems of bosons, for which

(B) for all $n, D_{n} U_{\pi}=D_{n}$ for any permutation operator $U_{\pi}$ on $\mathcal{H}^{\otimes n}$.

Condition (B) is stronger than (A). We also want the sequence $\left\{D_{n}\right\}$ of density operators to be consistent with respect to "subsampling" in the sense that

(C) for all $m<n, D_{n: m}=D_{m}$,

where $D_{n: m}$ denotes the $m^{t h}$ partial trace of $D_{n}$, i.e., the operator such that

$$
\operatorname{Tr}\left(D_{n: m} A\right)=\operatorname{Tr}\left(D_{n}(A \otimes I \stackrel{n-m \text { times }}{\otimes} \cdots \otimes \otimes)\right.
$$

for all $A \in \mathcal{B}\left(\mathcal{H}^{\otimes m}\right)$. 
The structure of sequences $\left\{D_{n}\right\}$ of density operators satisfying $(\mathrm{C})$ and (A) or (B) is given by the quantum analogue of the de Finetti Theorem of probability theory [2. Let $\rho$ be a density operator on $\mathcal{H}$. A sequence $\left\{D_{n}\right\}$ of density operators of the form

$$
\begin{aligned}
& D_{1}=\rho \\
& D_{2}=\rho \otimes \rho \\
& D_{3}=\rho \otimes \rho \otimes \rho, \quad \text { et cetera }
\end{aligned}
$$

always satisfies (A) and (C), but it satisfies (B) and (C) if and only if $\rho$ is a pure state, i.e., a rank one projector on $\mathcal{H}$. Roughly speaking, any sequence of density operators satisfying (A) and (C) is uniquely representable as a mixture of sequences of the form (2). That is, if $\left\{D_{n}\right\}$ satisfies (A) and (C) then there exists a unique probability measure $\mu$ supported on the singleparticle density operators such that

$$
D_{n}=\int \rho^{\otimes n} \mu(d \rho)
$$

for all $n$. Furthermore, if $\left\{D_{n}\right\}$ satisfies $(\mathrm{B})$ and $(\mathrm{C})$, then the measure $\mu(d \rho)$ in the integral representation (3) is even supported on the set of vector states $\rho=P_{\psi}$. This paraphrases the propositions of [2], ignoring the technical details; we now restate the results with more care.

For $m \leq n$, let $j_{m n}$ denote the ${ }^{*}$-isomorphic embedding

$$
j_{m n}(B)=B \otimes I^{\otimes n-m}
$$

of $\mathcal{B}\left(\mathcal{H}^{\otimes m}\right)$ into $\mathcal{B}\left(\mathcal{H}^{\otimes n}\right)$. The system of $\mathrm{C}^{*}$ algebras $\mathcal{B}\left(\mathcal{H}^{\otimes n}\right)$ and isomorphic injections $j_{m n}$ has an inductive limit $\mathcal{A}$. The inductive or direct limit in the category of $\mathrm{C}^{*}$ algebras may be constructed as in [3, Proposition 11.4.1]. The inductive limit $\mathcal{A}$ is unique up to isomorphism, and for each $n$ there is a ${ }^{*}$-isomorphism $i_{n}$ from $\mathcal{B}\left(\mathcal{H}^{\otimes n}\right)$ into $\mathcal{A}$ such that $i_{n} j_{m n}=i_{m}$ for all $m \leq n$ and the union of the images $i_{n}\left(\mathcal{B}\left(\mathcal{H}^{\otimes n}\right)\right)$ is dense in $\mathcal{A}$. A sequence $\left\{D_{n}\right\}$ of density operators satisfying the conditions (C) can be used to define a continuous positive linear functional $\omega$ on $\mathcal{A}$ by

$$
\omega\left(i_{n}(B)\right)=\operatorname{Tr}\left(D_{n} B\right) \quad \forall B \in \mathcal{B}\left(\mathcal{H}^{\otimes n}\right) .
$$

This is well-defined thanks to the consistency conditions $(\mathrm{C})$ and the density of $\cup i_{n}\left(\mathcal{B}\left(\mathcal{H}^{\otimes n}\right)\right)$ in $\mathcal{A}$. In particular, $\omega(e)=1$, where $e$ is the identity element 
of the $\mathrm{C}^{*}$ algebra $\mathcal{A}$. If $\left\{D_{n}\right\}$ satisfies $(\mathrm{A})$ as well as $(\mathrm{C})$ then $\omega$ is symmetric in the sense that

$$
\omega\left(i_{n}\left(U_{\pi} B U_{\pi}^{*}\right)\right)=\omega\left(i_{n}(B)\right)
$$

for all $n$, all $B \in \mathcal{B}\left(\mathcal{H}^{\otimes n}\right)$, and all $\pi \in \Pi_{n}$, the set of permutations of $\{1,2, \ldots, n\}$. The set of all "symmetric states" on $\mathcal{A}$, i.e., the set

$$
S S=\left\{\omega \in \mathcal{A}^{*} \mid \omega(e)=1 \text { and } \omega\left(x^{*} x\right) \geq 0 \forall x \in \mathcal{A} \text { and } \omega \text { satisfies (15) }\right\},
$$

is a convex subset of the Banach dual $\mathcal{A}^{*}$ of $\mathcal{A}$, and it is compact with respect to the weak* topology. Let $S S_{1}$ denote the space of single-particle states, i.e., the set

$$
S S_{1}=\left\{\rho \in \mathcal{B}(\mathcal{H})^{*} \mid \rho(I)=1 \text { and } \omega\left(A^{*} A\right) \geq 0 \forall A \in \mathcal{B}(\mathcal{H})\right\}
$$

endowed with the relative weak* topology it inherits as a subset of the Banach dual $\mathcal{B}(\mathcal{H})^{*}$ of $\mathcal{B}(\mathcal{H})$. It was first shown in [4] that each $\omega \in S S$ has a unique representation as an integral of product states

$$
\omega=\int_{S S_{1}} \rho \otimes \rho \otimes \rho \otimes \cdots \cdots \mu(d \rho)=\int_{S S_{1}} \rho^{\otimes \infty} \mu(d \rho),
$$

where $\mu$ is a probability measure on the $\sigma$-algebra $\mathcal{F}_{1}$ generated by the intersections with $S S_{1}$ of weak* open sets in $\mathcal{B}(\mathcal{H})^{*}$. We sketch a proof of this, following reference [2]: First, the extreme points of $S S$ are identified as the product states $\rho^{\otimes \infty}$. Thus, the set of extreme points is the image of the compact space $S S_{1}$ under the continuous injection $\rho \longmapsto \rho^{\otimes \infty}$, and it follows that the extreme set is closed in $S S$. The existence of an integral representation (6) is then a consequence of the Krein-Milman Theorem, and its uniqueness is shown in [2] by a direct argument.

It is further shown in 2] that the measure $\mu(d \rho)$ appearing in the integral representation (6) of $\omega$ is supported on the measurable subset of normal states on $\mathcal{B}(\mathcal{H})$ if $\omega$ is determined, as in formula (41) above, by sequences of density operators satisfying (A) and (C). If, in addition, the sequence of density operators defining $\omega$ satisfies $(\mathrm{B})$, then the measure $\mu(d \rho)$ is even supported on the vector states $\rho(A)=\langle\psi, A \psi\rangle$ with $\|\psi\|=1$.

\section{Examples of bosonic de Finetti states}

In this section we exhibit some sequences $\left\{D_{n}\right\}$ satisfying $(\mathrm{B})$ and $(\mathrm{C})$ that are obtained from natural statistical ensembles. In all of these examples, the 
single-particle Hilbert space $\mathcal{H}$ is finite dimensional. After introducing the notation, we will state all of our results before proceeding to their proofs.

Let $\mathcal{H}=\mathbb{C}^{d+1}$ and let $\mathcal{H}^{(n)}$ denote the subspace of symmetric vectors in $\mathcal{H}^{\otimes n}$. Let $\Sigma_{n}$ denote the symmetrizing projector

$$
\Sigma_{n}=\frac{1}{n !} \sum_{\pi \in \Pi_{n}} U_{\pi}
$$

from $\mathcal{H}^{\otimes n}$ onto $\mathcal{H}^{(n)}$. We now introduce notation for the occupation number basis of $\mathcal{H}^{(n)}$ relative to a fixed orthonormal (ordered) basis $\left\{e_{j}\right\}$ of $\mathcal{H}$. Let $\mathbf{n}=\left(n_{0}, n_{1}, \ldots, n_{d}\right)$ be an ordered $d+1$-tuple of nonnegative integers (occupation numbers) and let $\# \mathbf{n}$ denote $\sum n_{j}$. We use the notation

$$
\left(\begin{array}{l}
n \\
\mathbf{n}
\end{array}\right)=n ! / \prod_{i=0}^{d} n_{i} !
$$

for multinomial coefficients. The vector

$$
\Psi_{\mathbf{n}}=\sqrt{\left(\begin{array}{l}
n \\
\mathbf{n}
\end{array}\right)} \Sigma_{n}\left(e_{0}^{\otimes n_{0}} \otimes e_{1}^{\otimes n_{1}} \otimes \cdots \otimes e_{d}^{\otimes n_{d}}\right)
$$

is a unit vector in $\mathcal{H}^{(n)}$, and the set of vectors $\left\{\Psi_{\mathbf{n}} \mid \# \mathbf{n}=n\right\}$ is an orthonormal basis of $\mathcal{H}^{(n)}$. Let $P_{\mathbf{n}}$ denote the rank-one projector onto the span of $\Psi_{\mathbf{n}}$ :

$$
P_{\mathbf{n}} \Phi=\left\langle\Psi_{\mathbf{n}}, \Phi\right\rangle \Psi_{\mathbf{n}} .
$$

We begin by considering the "uniformly mixed" density operators supported on $\mathcal{H}^{(n)}$ :

Proposition 1 Let $\Sigma_{n}$ denote the symmetrizing projector (7). For each $m$,

$$
S_{m} \equiv \lim _{n \rightarrow \infty} \frac{1}{\operatorname{Tr} \Sigma_{n}} \Sigma_{n: m}=\sum_{\mathbf{m}: \# \mathbf{m}=m}\left\{\left(\begin{array}{l}
m \\
\mathbf{m}
\end{array}\right) \int_{\Delta_{d}} \prod_{i=0}^{d} p_{i}^{m_{i}} \lambda_{d}(d p)\right\} P_{\mathbf{m}}
$$

where $\lambda_{d}(d p)$ denotes normalized Lebesgue measure on the d-dimensional simplex

$\Delta_{d}=\left\{p=\left(p_{0}, p_{1}, \ldots, p_{d}\right) \in \mathbb{R}^{d+1} \mid 0 \leq p_{i} \quad i=1,2, \ldots, d \quad\right.$ and $\left.\quad \sum_{i=0}^{d} p_{i}=1\right\}$. 
The sequence $\left\{S_{m}\right\}$ satisfies (B) and (C) of Section 2, By the Quantum de Finetti Theorem, there exists a measure $\mu$ supported on the pure states on $\mathbb{C}^{d+1}$ such that

$$
S_{m}=\int P^{\otimes m} \mu(d P)
$$

for all $m \in \mathbb{N}$. This measure can be described as follows. Define the map

$$
\mathrm{v}: \Delta_{d} \times[0,2 \pi)^{d+1} \longrightarrow \mathbb{C}^{d+1}
$$

by

$$
\mathrm{v}\left(p_{0}, p_{1}, \ldots, p_{d}, \theta_{0}, \theta_{1}, \ldots, \theta_{d}\right)=\sum_{j=0}^{d} e^{i \theta_{j}} \sqrt{p_{j}} e_{j}
$$

where $\left\{e_{i}\right\}$ is the standard basis of $\mathbb{C}^{d+1}$. The map v is many-one onto the set of unit vectors in $\mathbb{C}^{d+1}$. The probability measure $\mu(d P)$ is the one induced via $\mathrm{v}$ from the uniform measure

$$
\lambda(d p) \sigma(d \theta) \equiv \lambda(d p) \frac{d \theta_{0}}{2 \pi} \frac{d \theta_{1}}{2 \pi} \cdots \frac{d \theta_{d}}{2 \pi}
$$

on $\Delta_{d} \times[0,2 \pi)^{d+1}$. In other words,

Proposition 2 The density operator (10) equals

$$
\int_{\Delta_{d}} \int_{[0,2 \pi)^{d+1}}\left(P_{\mathrm{v}(p, \theta)} \stackrel{m \text { times }}{\otimes} \cdots \otimes P_{\mathrm{v}(p, \theta)}\right) \sigma(d \theta) \lambda_{d}(d p) .
$$

Next we consider Gibbs ensembles for noninteracting systems of bosons. Let

$$
H_{n}=\sum_{i=1}^{n} T_{i}
$$

be the Hamiltonian for $n$ noninteracting bosons with single-particle space $\mathcal{H}=\mathbb{C}^{d+1}$. Let $\left\{e_{j}\right\}$ be an orthonormal basis of $\mathcal{H}$ consisting of eigenvectors of the single-particle operator $T$, so that $T e_{j}=\epsilon_{j} e_{j}$. The Gibbs density operator for the $n$ boson system is

$$
\Gamma_{n}(\beta)=\frac{1}{Z_{n, \beta}} \sum_{\mathbf{n}: \# \mathbf{n}=n} \prod_{i=0}^{d} e^{-\beta n_{i} \epsilon_{i}} P_{\mathbf{n}} \quad \text { with } \quad Z_{n, \beta}=\sum_{\mathbf{n}: \# \mathbf{n}=n} \prod_{i=0}^{d} e^{-\beta n_{i} \epsilon_{i}} .
$$

An interesting limit is attained if temperature is scaled in proportion to $n$ as $n \longrightarrow \infty$. If the temperature is not scaled as $n \longrightarrow \infty$ then a sort of Bose-Einstein condensation is attained in the limit. 
Proposition 3 Let $H_{n}$ be the noninteracting Hamiltonian (13) and let $\Gamma_{n}(\beta)$ denote the Gibbs density (14). Let $\left\{e_{j}\right\}$ be an orthonormal basis of $\mathcal{H}$ consisting of eigenvectors of the single-particle operator $T$, so that $T e_{j}=\epsilon_{j} e_{j}$.

(i) For each $m \in \mathbb{N}$, the limit $\lim _{n \rightarrow \infty} \Gamma_{n: m}(\beta / n)$ exists and equals

$$
\sum_{\mathbf{m}: \# \mathbf{m}=m}\left\{\left(\begin{array}{c}
m \\
\mathbf{m}
\end{array}\right) \int_{\Delta_{d}} \prod_{i=0}^{d} p_{i}^{m_{i}} Z_{\beta}^{-1} \prod_{i=0}^{d} e^{-\beta \epsilon_{i} p_{i}} \lambda_{d}(d p)\right\} P_{\mathbf{m}}
$$

with $Z_{\beta}^{-1}=\int_{\Delta_{d}} \prod_{i=0}^{d} \exp \left(-\beta \epsilon_{i} p_{i}\right) \lambda_{d}(d p)$.

(ii) If $\epsilon_{0}<\epsilon_{1} \leq \cdots \leq \epsilon_{d}$, then for each $m \in \mathbb{N}$,

$$
\lim _{n \rightarrow \infty} \Gamma_{n: m}(\beta)=P_{(m, 0, \ldots, 0)}=P_{e_{0}}^{\otimes m} .
$$

Finally, we consider systems with two-particle interactions in the "mean field" scaling. Let $V$ be a Hamiltonian operator on $\mathcal{H} \otimes \mathcal{H}$ such that $V(x \otimes$ $y)=V(y \otimes x)$ for all $x, y \in \mathcal{H}$. For $n>2$, define the Hamiltonian

$$
H_{n}=\sum_{i=1}^{n} T_{i}+\frac{1}{n-1} \sum_{1 \leq i<j \leq n} V_{i j}
$$

where $V_{i j}$ denotes the operator obtained by applying $V$ to the $i^{\text {th }}$ and $j^{\text {th }}$ factors of $\mathcal{H}^{\otimes n}$. For any $n \in \mathbb{N}$ and any $\beta \in \mathbb{R}$, the $n$-particle Gibbs density at inverse temperature $\beta$ for the Hamiltonian (15) is

$$
\Gamma_{n}(\beta)=\frac{1}{\operatorname{Tr}\left(e^{\left.-\beta H_{n} \Sigma_{n}\right)}\right.} e^{-\beta H_{n}} \Sigma_{n} .
$$

Proposition 4 Let $\Gamma_{n}(\beta)$ denote the Gibbs density (16). For each $m$, the limit

$$
G_{m}=\lim _{n \rightarrow \infty}\left\{\Gamma_{n}(\beta / n)\right\}_{: m}
$$

exists and defines a density operator on $\left(\mathbb{C}^{d+1}\right)^{\otimes n}$. The de Finetti representation of $G_{m}$ is

$$
\frac{1}{Z_{\beta}} \int_{\Delta_{d}} \int_{[0,2 \pi)^{d+1}} P_{\mathrm{v}} \stackrel{m \text { times }}{\otimes \cdots \otimes P_{\mathrm{v}}} e^{-\beta\left\{\operatorname{Tr}\left(T P_{\mathrm{v}}\right)+\operatorname{Tr}\left(V\left(P_{\mathrm{v}} \otimes P_{\mathrm{v}}\right)\right) / 2\right\}} \sigma(d \theta) \lambda_{d}(d p)
$$

with $\mathrm{v}=\mathrm{v}(p, \theta)$ as in (11) and

$$
Z_{\beta}=\int_{\Delta_{d}} \int_{[0,2 \pi)^{d+1}} e^{-\beta\left\{\operatorname{Tr}\left(T P_{\mathrm{v}}\right)+\operatorname{Tr}\left(V\left(P_{\mathrm{v}} \otimes P_{\mathrm{v}}\right)\right) / 2\right\}} \sigma(d \theta) \lambda_{d}(d p) .
$$


The rest of this section is devoted to proving the above propositions.

Recall the definition (9) of the projectors $P_{\mathbf{n}}$. For each $n \in \mathbb{N}$, let $\rho_{n}$ be an $n$-particle density

$$
\rho_{n}=\sum_{\mathbf{n}: \# \mathbf{n}=n} w_{n}(\mathbf{n}) P_{\mathbf{n}}
$$

where $w_{n}$ is a probability measure on the set $\{\mathbf{n} \mid \# \mathbf{n}=n\}$. Each probability measure $w_{n}$ can be associated with the discrete probability measure

$$
\omega_{n}=\sum_{\mathbf{n}: \# \mathbf{n}=n} w_{n}(\mathbf{n}) \delta\left(p-\frac{1}{n} \mathbf{n}\right)
$$

on the $d$-dimensional simplex $\Delta_{d}$. It may be verified that

$$
P_{\mathbf{n}: m}=\left(\begin{array}{c}
n \\
m
\end{array}\right)^{-1} \sum_{\mathbf{m}: \# \mathbf{m}=m} \prod_{i=0}^{d}\left(\begin{array}{c}
n_{i} \\
m_{i}
\end{array}\right) P_{\mathbf{m}}
$$

(this equals 0 if any $m_{i}>n_{i}$ for any $i$ ), and therefore

$$
\begin{aligned}
\rho_{n: m} & =\left(\begin{array}{c}
n \\
m
\end{array}\right)^{-1} \sum_{\mathbf{m}: \# \mathbf{m}=m}\left[\sum_{\mathbf{n}: \# \mathbf{n}=n} w_{n}(\mathbf{n}) \prod_{i=0}^{d}\left(\begin{array}{c}
n_{i} \\
m_{i}
\end{array}\right)\right] P_{\mathbf{m}} \\
& =\sum_{\mathbf{m}: \# \mathbf{m}=m}\left(\begin{array}{c}
m \\
\mathbf{m}
\end{array}\right)\left[\sum_{\mathbf{n}: \# \mathbf{n}=n} w_{n}(\mathbf{n}) \frac{\prod_{i=0}^{d} \frac{n_{i}}{n}\left(\frac{n_{i}}{n}-\frac{1}{n}\right) \cdots\left(\frac{n_{i}}{n}-\frac{m_{i}-1}{n}\right)}{1\left(1-\frac{1}{n}\right)\left(1-\frac{2}{n}\right) \cdots\left(1-\frac{m-1}{n}\right)}\right] P_{\mathbf{m}} .
\end{aligned}
$$

The coefficient of $P_{\mathbf{m}}$ in (17) may be written

$$
\left(\begin{array}{l}
m \\
\mathbf{m}
\end{array}\right) \int_{\Delta_{d}} f_{n}(p) \omega_{n}(d p)
$$

where

$$
f_{n}(p)=\mathbb{1}_{\left\{p_{i}>\left(m_{i}-1\right) / n \forall i\right\}}(p) \frac{\prod_{i=0}^{d} p_{i}\left(p_{i}-\frac{1}{n}\right) \cdots\left(p_{i}-\frac{m_{i}-1}{n}\right)}{1\left(1-\frac{1}{n}\right)\left(1-\frac{2}{n}\right) \cdots\left(1-\frac{m-1}{n}\right)} .
$$

The functions $f_{n}(p)$ converge uniformly to $\prod_{i=0}^{d} p_{i}^{m_{i}}$ on $\Delta_{d}$. Therefore, if $\omega_{n}$ converges weakly to some probability measure $\omega(d p)$ on $\Delta_{d}$, then

$$
\lim _{n \rightarrow \infty} \rho_{n: m}=\sum_{\mathbf{m}: \# \mathbf{m}=m}\left(\begin{array}{l}
m \\
\mathbf{m}
\end{array}\right) \int_{\Delta_{d}} \prod_{i=0}^{d} p_{i}^{m_{i}} \omega(d p) P_{\mathbf{m}} .
$$


The probability measures on $\Delta_{d}$ corresponding to the Gibbs density operators (14) for noninteracting bosons are

$$
\omega_{n}=Z_{n, \beta}^{-1} \sum_{\mathbf{n}: \# \mathbf{n}=n} \prod_{i=0}^{d} e^{-\beta n_{i} \epsilon_{i}} \delta\left(p-\frac{1}{n} \mathbf{n}\right) .
$$

If all of the eigenvalues of $T$ are equal, then the measures (19) converge weakly to $\lambda_{d}(d p)$, the uniform probability measure on the simplex, but if $\epsilon_{0}$ is strictly smaller than all of the other eigenvalues of $T$, then the measures (19) converge weakly to $\delta(p-(1,0, \ldots, 0))$, a point-mass at the lowest energy vertex of the simplex. This convergence implies Propositions 1 and assertion (ii) of Proposition 3 by formula (18). On the other hand, the probability measures corresponding to the Gibbs density operators $\Gamma_{n}(\beta / n)$ for noninteracting bosons are

$$
\omega_{n}=Z_{n, \beta}^{-1} \sum_{\mathbf{n}: \# \mathbf{n}=n} \prod_{i=0}^{d} e^{-\beta \epsilon_{i} n_{i} / n} \delta\left(p-\frac{1}{n} \mathbf{n}\right)
$$

and these converge weakly to

$$
Z_{\beta}^{-1} \prod_{i=0}^{d} e^{-\beta \epsilon_{i} p_{i}} \lambda_{d}(d p)
$$

with $Z_{\beta}=\int_{\Delta_{d}} \prod_{i=0}^{d} \exp \left(-\beta \epsilon_{i} p_{i}\right) \lambda_{d}(d p)$. This proves assertion (i) of Proposition 3 .

To prove Proposition 2, we will show that (12) and (10) are equal. Define the rank-one operators $Q_{j k}(x)=\left\langle e_{k}, x\right\rangle e_{j}$. From (11),

$$
P_{\mathrm{v}(p, \theta)}=\sum_{j, k=0}^{d} e^{i\left(\theta_{j}-\theta_{k}\right)} \sqrt{p_{j} p_{k}} Q_{j k}
$$

and therefore $P_{\mathrm{v}(p, \theta)}{ }^{\otimes m}$ equals

$$
\sum_{j_{1}, \ldots, j_{m}=0}^{d} \sum_{k_{1}, \ldots, k_{m}=0}^{d} \prod_{r=0}^{d} \sqrt{p_{j_{r}} p_{k_{r}}} e^{i\left(\theta_{j_{r}}-\theta_{k_{r}}\right)} Q_{j_{1} k_{1}} \otimes Q_{j_{2} k_{2}} \otimes \cdots \otimes Q_{j_{m} k_{m}}
$$

For $i=0,1, \ldots, d$, let $N_{i}:\{0,1, \ldots, d\}^{m} \longrightarrow \mathbb{N}$ be defined by

$$
N_{i}\left(x_{1}, x_{2}, \ldots, x_{m}\right)=\#\left\{k \in\{1,2, \ldots, m\}: x_{k}=i\right\}
$$


and define

$$
N\left(x_{1}, x_{2}, \ldots, x_{m}\right)=\left(N_{0}\left(x_{1}, x_{2}, \ldots, x_{m}\right), \ldots, N_{d}\left(x_{1}, x_{2}, \ldots, x_{m}\right)\right) .
$$

If $N\left(j_{1}, \ldots, j_{m}\right)=N\left(k_{1}, \ldots, k_{m}\right)$ then

$$
\int_{[0,2 \pi)^{d+1}} \prod_{r=0}^{d} e^{i\left(\theta_{j_{r}}-\theta_{k_{r}}\right)} \sigma(d \theta)=1,
$$

but otherwise it equals 0. Thus, from (20),

$$
\begin{aligned}
& \int_{\Delta_{d}} \int_{[0,2 \pi)^{d+1}} P_{\mathrm{v}(p, \theta)}{ }^{\otimes m} \sigma(d \theta) \lambda_{d}(d p) \\
= & \sum_{\mathbf{m}: \# \mathbf{m}=m} \int_{\Delta_{d}} \prod_{i=0}^{m} p_{i}^{m_{i}} \lambda_{d}(d p) \sum_{\substack{j_{(j,}, \ldots, j_{m}: \\
\left(j_{1}, \ldots, j_{m}\right)=\mathbf{m}}} \sum_{\substack{k_{1}, \ldots, k_{m}: \\
\left(k_{1}, \ldots, k_{m}\right)=\mathbf{m}}} Q_{j_{1} k_{1}} \otimes \cdots \otimes Q_{j_{m} k_{m}} \\
= & \sum_{\mathbf{m}: \# \mathbf{m}=m} \int_{\Delta_{d}} \prod_{i=0}^{m} p_{i}^{m_{i}} \lambda_{d}(d p)\left(\begin{array}{c}
m \\
\mathbf{m}
\end{array}\right) P_{\mathbf{m}}
\end{aligned}
$$

by the definition (9) of $P_{\mathbf{m}}$. This proves Proposition 2 ,

Finally, we derive Proposition 4 from Proposition 2, Define $W=T \otimes I+$ $I \otimes T+V$. Then the Hamiltonian (15) can be written

$$
H_{n}=\frac{1}{n-1} \sum_{1 \leq i<j \leq n} W_{i j} .
$$

We claim that

$$
\lim _{n \rightarrow \infty} \frac{n^{-j}}{\operatorname{Tr} \Sigma_{n}}\left\{\left(H_{n}\right)^{j} \Sigma_{n}\right\}_{: m}=2^{-j}\left\{W_{m+1, m+2} W_{m+3, m+4} \cdots W_{m+2 j-1, m+2 j} S_{m+2 j}\right\}_{: m}
$$

for each $j, m \in \mathbb{N}$. This is so because $\left(H_{n}\right)^{j}$ contains $\left(\begin{array}{c}n \\ 2\end{array}\right)^{j}$ terms of the form $(n-1)^{-j} W_{a_{1} b_{1}} W_{a_{2} b_{2}} \cdots W_{a_{j} b_{j}}$, and, when $n$ is large, the majority of these terms are such that the indices $a_{1}, b_{1}, \ldots, a_{j}, b_{j}$ are all distinct and greater than $m$. The sum of the remaining terms in $\left(H_{n}\right)^{j}$ is $o\left(n^{j}\right)$ and does not contribute to the limit (21). By the symmetry of $\Sigma_{n}$,

$$
\begin{aligned}
\left\{W_{a_{1} b_{1}} W_{a_{2} b_{2}} \cdots W_{a_{j} b_{j}} \Sigma_{n}\right\}_{: m} & =\left\{W_{m+1, m+2} \cdots W_{m+2 j-1, m+2 j} \Sigma_{n}\right\}_{: m} \\
& =\left\{W_{m+1, m+2} \cdots W_{m+2 j-1, m+2 j} \Sigma_{n: m+2 j}\right\}_{: m}
\end{aligned}
$$


if $a_{1}, b_{1}, \ldots, a_{j}, b_{j}$ are all distinct and greater than $m$. There are asymptotically $n^{2 j} / 2$ such terms, so (21) follows from Proposition 1]

Now, to prove Proposition 44 expand

$$
\frac{1}{\operatorname{Tr} \Sigma_{n}} e^{-\beta n^{-1} H_{n}} \Sigma_{n}=\sum_{j=0}^{\infty} \frac{1}{j !}(-\beta)^{j} n^{-j}\left(H_{n}\right)^{j} \frac{1}{\operatorname{Tr} \Sigma_{n}} \Sigma_{n}
$$

and take the $m^{\text {th }}$ partial trace:

$$
\frac{1}{\operatorname{Tr} \Sigma_{n}}\left\{e^{-\beta n^{-1} H_{n}} \Sigma_{n}\right\}_{: m}=\sum_{j=0}^{\infty} \frac{1}{j !}(-\beta)^{j} n^{-j}\left\{\left(H_{n}\right)^{j} \frac{1}{\operatorname{Tr} \Sigma_{n}} \Sigma_{n}\right\}_{: m} .
$$

The $j^{\text {th }}$ term of the series in (22) converges to

$$
(-1)^{j} \frac{1}{j !}\left(\frac{\beta}{2}\right)^{j}\left\{W_{m+1, m+2} W_{m+3, m+4} \cdots W_{m+2 j-1, m+2 j} S_{m+2 j}\right\}_{: m}
$$

as $n \longrightarrow \infty$ by (21) and is bounded by $\frac{1}{j !} \beta^{j}\|W\|^{j}$ uniformly in $n$. Since the series in (22) are majorized by the convergent series $\sum_{j} \frac{1}{j !} \beta^{j}\|W\|^{j}$ and converge term-by-term as $n \longrightarrow \infty$, it follows that

$\lim _{n \rightarrow \infty} \frac{1}{\operatorname{Tr} \Sigma_{n}}\left\{e^{-\beta n^{-1} H_{n}} \Sigma_{n}\right\}_{: m}=\sum_{j=0}^{\infty}(-1)^{j} \frac{1}{j !}\left(\frac{\beta}{2}\right)^{j}\left\{W_{m+1, m+2} \cdots W_{m+2 j-1, m+2 j} S_{m+2 j}\right\}_{: m}$.

Substituting the integral representations (12) for $S_{m+2 j}$ into (23) yields

$$
\begin{aligned}
& \lim _{n \rightarrow \infty} \frac{1}{\operatorname{Tr} \Sigma_{n}}\left\{e^{-\beta n^{-1} H_{n}} \Sigma_{n}\right\}_{: m} \\
& =\sum_{j=0}^{\infty} \frac{1}{j !}\left(\frac{-\beta}{2}\right)^{j} \int_{\Delta_{d}} \int_{[0,2 \pi)^{d+1}}\left[\operatorname{Tr}\left(W P_{\mathrm{v}(p, \theta)}{ }^{\otimes 2}\right)\right]^{j} P_{\mathrm{v}(p, \theta)}{ }^{\otimes m} \sigma(d \theta) \lambda_{d}(d p) \\
& =\int_{\Delta_{d}} \int_{[0,2 \pi)^{d+1}} e^{-\beta \frac{1}{2} \operatorname{Tr}\left(W P_{\mathrm{v}(p, \theta)} \otimes P_{\mathrm{v}(p, \theta)}\right)} P_{\mathrm{v}(p, \theta)} \otimes m \sigma(d \theta) \lambda_{d}(d p) .
\end{aligned}
$$

Proposition 4 follows from the preceding equation and the definition (16) of $\Gamma_{n}(\beta)$. 


\section{Acknowledgments}

I would like to thank Lucien Le Cam for listening very patiently to some of this story and for his kind encouragement. This work was supported by the Austrian START project "Nonlinear Schrödinger and quantum Boltzmann equations" of Norbert J. Mauser (contract Y-137-Tec).

\section{References}

[1] M. Fannes, H. Spohn and A. Verbeure. Equilibrium states for mean field models. J. Math. Physics 21(2), 355 - 358 (1980)

[2] R.L. Hudson and G.R. Moody. Locally normal symmetric states and an analogue of de Finetti's theorem. Z. für Wahrscheinlichkeit und verw. Geb. 33, 343-351 (1976)

[3] R.V. Kadison and J.R. Ringrose. Fundamentals of the Theory of Operator Algebras II. American Mathematical Society (1997)

[4] E. Størmer. Symmetric states of infinite tensor products of $\mathrm{C}^{*}$-algebras. Journal of Functional Analysis 3, 48 - 68 (1969) 